\title{
Effet de la nature du fourrage utilisé pendant la période d'élevage sur la digestibilité des fourrages à l'âge adulte chez les caprins et les ovins
}

\author{
PE Arista, ZM Bicaba, F Faurie, JL Tisserand \\ ENSSAA-INRA, laboratoire de recherches de la chaire de zootechnie, \\ 26, bd Docteur-Petitjean, 21000 Dijon, France
}

\begin{abstract}
Summary - Effect of forage quality during the rearing period on forage digestibility in adult sheep and goats. A study conducted on 20 lambs and 20 kids raised on diets based either on alfalfa hay or straw showed there was no real difference between the adult animals as regards their digestion of forage. However, the goats raised on alfalfa hay seemed to make better use of the nitrogen in the forages whereas the opposite was noticed in the case of sheep.
\end{abstract}

L'utilisation digestive comparée chez les ovins et les caprins est étudiée depuis longtemps (Ademosum, 1970; Huston, 1978; Cabrera et al, 1983; Tisserand et al, 1986). L'ensemble des travaux concluent à une meilleure digestion chez les caprins lorsqu'il s'agit de fourrages pauvres. Par contre, avec des fourrages de qualité moyenne à bonne, il ne semble pas y avoir de différences, et, parfois même, les ovins sont plus performants que les caprins. Malgré ces résultats, plusieurs points restent à préciser, notamment les effets de la nature de l'alimentation utilisée pendant la période d'élevage sur la capacité des animaux à utiliser différents régimes.

Matériel et méthodes - Vingt agneaux et 20 chevreaux, achetés à l'âge de $8 \mathrm{j}$, sont élevés en un seul groupe par espèce pendant 4 semaines au cours desquelles l'alimentation est à base de lait de remplacement. À la fin de cette période, les animaux de chaque espèce sont répartis en 2 lots sur la base de leur poids vif et de leur gain de poids pendant la période. Deux lots (un par espèce) sont alimentés avec un régime constitué de foin de luzerne + aliment concentré (maïs + tourteau de soja) : régime $F$, alors que les 2 autres lots reçoivent un régime constitué de paille de blé + aliment concentré (maïs + tourteau de soja) : régime P. Au bout de 18 mois, 3 animaux (mâles castrés) de chaque lot pesant $53 \pm 5 \mathrm{~kg}$ pour les caprins et $75 \pm 5 \mathrm{~kg}$ pour les ovins, sont placés dans des cages à digestibilité. Pendant une première période expérimentale de 4 semaines, ils reçoivent le même régime que celui distribué pendant la période d'élevage : soit $65 \%$ de paille $+35 \%$ de concentré, soit $80 \%$ de foin de luzerne $+20 \%$ de concentré. Pendant une $2^{e}$ période de même durée, les régimes sont permutés. Le concentré distribué avec le foin est composé de $76 \%$ de maïs et $14 \%$ de tourteau de soja pour les ovins, et de $100 \%$ de maïs pour les caprins; avec la paille, il est composé de $50 \%$ de maïs et $50 \%$ de tourteau de soja pour les ovins, et de $56 \%$ de maïs et $44 \%$ de tourteau de soja pour les caprins. Les rations sont distribuées 2 fois par jour, en quantité limitée aux besoins. La digestibilité de la ration (matière organique - $\mathrm{MO}-$, azote $-\mathrm{N}-$, cellulose brute - $\mathrm{CB}-$ ) a été mesurée par collecte totale des fèces, à chaque période, au cours des 10 derniers jours. Les résultats sont soumis à l'analyse de variance et au test $t$ de Student. 
Résultats et discussion - Quels que soient l'espèce et le régime d'élevage des animaux, la digestibilité de la MO et de la CB est peu différente avec le régime expérimental foin de luzerne + concentré. Les ovins élevés avec le régime $P$ ont une meilleure digestibilité de l'azote que ceux élevés avec le régime $F(P<0,01)$. La tendance est la même chez les caprins. En revanche, avec le régime expérimental paille de blé + concentré, les digestibilités de la MO et de la CB sont plus élevées chez les caprins que chez les ovins $(P<0,01)$; quels qu'aient été les régimes d'élevage. Entre les animaux de la même espèze, les digestibilités de la MO et de la $\mathrm{CB}$ ne sont pas différentes, mais celle de l'azote est nettement plus élevée chez les caprins dont le régime d'élevage était le régime $F$ que chez ceux élevés avec le régime $P(P<$ $0,01)$. Pour les 2 régimes, il n'est pas possible de comparer la digestibilité de l'azote entre les ovins et les caprins, la teneur en $\mathrm{N}$ des régimes étant différente.

En conclusion, il semblerait donc que, dans le cas des caprins, le fourrage de bonne qualité utilisé pendant la période d'élevage joue un rôle favorable dans la digestibilité de l'azote du régime paille de blé + concentré. Par contre, dans le cas des ovins, le fourrage de bonne qualité semble avoir un effet inverse. Cependant, ces résultats devront être confirmés car on constate de fortes variations individuelles dans les lots expérimentaux, surtout dans le cas des caprins.

Ademosum AA (1970) Niger Agric J 7, 119 Cabrera R, Villareal P, Viale Castillo (1983) $J$ Anim Sci 13, 213-219

Huston JE (1978) J Dairy Sci 61, 988-993

Tisserand JL, Bellet B, Masson C (1986) Reprod Nutr Dév 26 (1B), 313-314

Tableau I. Digestibilité (\%) des régimes.

\begin{tabular}{lccccc}
\hline $\begin{array}{l}\text { Régime } \\
\text { expérimental }\end{array}$ & $\begin{array}{c}\text { Espèce } \\
\text { animale }\end{array}$ & $\begin{array}{c}\text { Régime } \\
\text { d'élevage }\end{array}$ & $\begin{array}{c}\text { Matière } \\
\text { organique }\end{array}$ & Azote & $\begin{array}{c}\text { Cellulose } \\
\text { brute }\end{array}$ \\
\hline Foin de luzerne & $\mathrm{O}$ & $\mathrm{F}$ & $63 \pm 1$ & $63 \pm 1$ & $48 \pm 2$ \\
+ concentré & $\mathrm{O}$ & $\mathrm{P}$ & $64 \pm 2$ & $67 \pm 1^{\mathrm{a}}$ & $47 \pm 3$ \\
& $\mathrm{C}$ & $\mathrm{F}$ & $62 \pm 3$ & $56 \pm 4$ & $50 \pm 1$ \\
& $\mathrm{C}$ & $\mathrm{P}$ & $61 \pm 3$ & $63 \pm 6$ & $45 \pm 8$ \\
Paille de blé & $\mathrm{O}$ & $\mathrm{F}$ & $49 \pm 3$ & $56 \pm 1$ & $34 \pm 6$ \\
+ concentré & $\mathrm{O}$ & $\mathrm{P}$ & $52 \pm 3$ & $53 \pm 2$ & $31 \pm 2$ \\
& $\mathrm{C}$ & $\mathrm{F}$ & $59 \pm 3^{\mathrm{a}}$ & $63 \pm 8^{\mathrm{a}}$ & $39 \pm 4^{\mathrm{a}}$ \\
& $\mathrm{C}$ & $\mathrm{P}$ & $56 \pm 3^{\mathrm{a}}$ & $53 \pm 1$ & $38 \pm 4^{\mathrm{a}}$ \\
\hline
\end{tabular}

a Différence ANOVA $(P<0,01)$. 